\title{
1 Colletotrichum spp. from soybean cause disease on lupin but can also induce 2 plant growth-promoting effects
}

4 Louisa Wirtz ${ }^{1}$, Nelson Sidnei Massola Júnior ${ }^{2}$, Renata Rebellato Linhares de Castro ${ }^{2}$, Brigitte

5 Ruge-Wehling ${ }^{3}$, Ulrich Schaffrath ${ }^{1}$, and Marco Loehrer ${ }^{1 \#}$

6

$7 \quad{ }^{1}$ Department of Plant Physiology, RWTH Aachen University, 52056 Aachen, Germany

$8{ }^{2}$ University of São Paulo, ESALQ, Department of Plant Pathology and Nematology, 9 Piracicaba/SP, Brazil

$10{ }^{3}$ Institute for Breeding Research on Agricultural Crops, Julius Kühn $\square$ Institut, Groß Lüsewitz, 11 Germany

12 \# corresponding author: loehrer@ bio3.rwth-aachen.de, Tel.: +49 2418020101

14 ORCID IDs:

15 Nelson Sidnei Massola Júnior: 0000-0001-8890-4773

16 Renata Rebellato Linhares: 0000-0001-9488-7164

17 Ulrich Schaffrath: 0000-0002-3691-0888

Marco Loehrer: 0000-0002-3740-0855 


\section{Abstract (150-200 words)}

23 Protein crop plants such as soybean and lupin attract increasing attention because of their potential use as forage, green manure or for the production of oil and protein for human consumption. While soybean production only recently gained more importance in Germany and within the whole EU in frame of protein strategies, lupin production already is well established in Germany. The cultivation of lupins is impeded by the hemibiotrophic ascomycete Colletotrichum lupini, the causing agent of anthracnose disease. Worldwide, soybean is also a host for a variety of Colletotrichum species, but so far this seems not to be the case in Germany. Cross-virulence between lupin and soybean infecting isolates is a potential threat, especially taking into consideration the overlap of possible soybean and lupine growing areas in Germany. To address this question, we systematically investigated the interaction of different Colletotrichum species isolated from soybean in Brazil on actual German soybean and lupin plant cultivars. Conversely, we tested the interaction of a German field isolate of $C$. lupini with soybean. Under controlled conditions, Colletotrichum species from soybean and lupin were able to cross-infect the other host plant with varying degrees of virulence, thus underpinning the potential risk of increased anthracnose diseases in the future. Interestingly, we observed a pronounced plant growth-promoting effect for some hostpathogen combinations which might open the route to the use of beneficial biological agents in lupine and soybean production.

\section{Keywords, 4-6} promoting effect 


\section{$47 \quad$ Introduction}

48 In view of the steadily growing world population and the increasing demand for sustainable

49 food production, it becomes clear that dietary protein cannot be provided through animal

50 products alone. Soybean is the primary source for non-animal protein and the most cultivated

51 protein-crop plant on a global scale. In Europe soybean and soybean-derived products are

52 imported for the most part (Lucas et al. 2015). Because European consumers shift their

purchase behavior more and more to non-GMO products from sustainable production soybean

imports from abroad are under debate. Hence, the independent production of protein-crop

plants in Germany becomes increasingly attractive, and lupin represents an appealing

alternative to soybean (Lucas et al. 2015; Talhinhas et al. 2016).

In the past decades, lupin production was negatively affected by the hemibiotrophic

Talhinhas et al. 2016; Pecchia et al. 2019). The influence of anthracnose epidemics was

severe, partially due to focusing on breeding of low-alkaloid containing white lupin in the

past, which is especially susceptible to C. lupini (Berger et al. 2012). Breeding for resistance

of lupin against $C$. lupini is slowly progressing, but still the impact of the disease on

production success is severe and alternative plant protection methods are needed (Fischer et

al. 2015; Jacob et al. 2017).

Currently, soybean cultivation in Germany is mostly affected by the fungal diseases

Sclerotinia sclerotiorum causing stem rot ("white mold") and the disease complex Diaporthe spp./Phomopsis spp, causing seed and stem blight (Ploper and Backman 1992; Murphysoybean production and because of the increasing production, it is only a matter of time until anthracnose will become a severe problem on soybean in Germany. 
71 Recently the causal agents of anthracnose on soybean were systematically investigated in

72 Brazil, describing C. truncatum as the major anthracnose-causing species but also identifying

73 C. plurivorum as a novel species capable of causing anthracnose (Rogério et al. 2017, 2019;

74 Barbieri et al. 2017; Dias et al. 2018; Damm et al. 2019).

75 In this study, we performed inoculation experiments with Colletotrichum species isolated

76 from soybean and lupin in Brazil and Germany, respectively, on plant cultivars used in

77 Germany to evaluate the risk of cross-infection in a scenario of increased legume production.

78 Results from this study shall contribute to planning of current and future plant breeding goals and open the road to novel plant protection strategies.

81 Material and Methods

\section{Plant material and growth conditions}

Seeds of Glycine max cv. Abelina (not inoculated with rhizobia) and Lupinus angustifolius cv. grown in a climate chamber (day/night cycle: $16 \mathrm{~h}$ light, $350 \mu \mathrm{mol} \mathrm{m} \mathrm{s}^{-2} \mathrm{PAR}, 24{ }^{\circ} \mathrm{C}, 65 \%$

$\mathrm{RH} ; 8$ h dark, $20{ }^{\circ} \mathrm{C}, 80 \% \mathrm{RH}$ ) in ED73 substrate (Balster Einheitserdewerk GmbH,

87 Fröndenberg, Germany).

\section{Fungal isolates and culture conditions}

The German field isolate of Colletotrichum lupini BBA70358 (=CBS 109222) is deposited at the CBS collection of the Westerdijk Fungal Biodiversity Institute (Utrecht, The Netherlands) and was donated to B. Ruge-Wehling by H. I. Nirenberg. C. truncatum isolates (see table 1) were provided by N.S. Massola Jr. and (University of São Paulo, ESALQ, Department of Plant Pathology and Nematology, Piracicaba/SP, Brazil ) stored at the culture collection of 
Paulo, Brazil (Rogério et al. 2017). Isolates of C. plurivorum (table 1) were also provided by

N.S. Massola Jr. and stored at the culture collection of Plant Pathology Department of 'Luiz de Queiroz' College of Agriculture, University of São Paulo, Brazil (Barbieri et al. 2017).

Richard O’Connell (BIOGER, INRA, Paris, France) kindly provided C. higginsianum IMI 349063. All fungi were propagated on PDA medium (potato extract glucose agar, Carl Roth

$\mathrm{GmbH}+\mathrm{Co} . \mathrm{KG}$, Karlsruhe, Germany). The fungal cultures were incubated at $25^{\circ} \mathrm{C}$ with a 12 h day/night cycle under cool white fluorescent lamps. Every 7-10 days fungal isolates were transferred to fresh PDA plates. To prevent degeneration of isolates (i. e. reduced sporulation), at regular intervals (every three months) material from stocks was used to start a new culture.

Table 1 - fungal isolates used in this study

\begin{tabular}{|l|l|l|}
\hline species & strain & isolated from \\
\hline Colletotrichum lupini & BBA70358 (=CBS109222) & \\
\hline Colletotrichum truncatum & LFN150 & lupin \\
\hline Colletotrichum truncatum & LFN151 & soybean \\
\hline Colletotrichum truncatum & LFN152 & soybean \\
\hline Colletotrichum truncatum & LFN153 & soybean \\
\hline Colletotrichum truncatum & LFN154 & soybean \\
\hline Colletotrichum plurivorum & LFN0007 & soybean \\
\hline Colletotrichum plurivorum & LFN0008 & soybean \\
\hline Colletotrichum plurivorum & LFN0010 (=URM7540) & soybean \\
\hline Colletotrichum plurivorum & LFN0018 (=URM7541) & soybean \\
\hline Colletotrichum plurivorum & LFN0019 (=URM7542) & soybean \\
\hline Colletotrichum plurivorum & LFN0023 & soybean \\
\hline Colletotrichum higginsianum & IMI349063 (,063A“) & soybean \\
\hline
\end{tabular}

\section{Hypocotyl assay}

The protocol for the toothpick inoculation assay was adapted from Scandiani et al. (2011). To prepare hypocotyls of soybean and lupin, seeds were placed on wet filter paper and incubated in the dark. Seeds that germinated after 2-3 days were planted in ED73 soil and placed in a climate chamber as described above. The hypocotyls were ready for the assay at the plant growth stage of formation of the first leaf. Sterile toothpick tips $(1.0-1.5 \mathrm{~cm})$ were placed 
around a growing fungal colony on PDA plates and used for hypocotyl inoculation when fully overgrown by fungal mycelium. The inoculated toothpick tips were pushed into hypocotyls using sterile forceps. After seven days lesion length was measured using a Leica MZ125 stereo-microscope equipped with a digital JVC KYF 750 camera using Diskus software (Technisches Büro Hilgers, Köngiswinter, Germany).

\section{Seed inoculation assay}

Soybean and lupin seeds were surface sterilized by washing in $70 \% \mathrm{EtOH}$ for 1', followed by

$3 \mathrm{x}$ rinsing in sterile distilled water. Afterwards, the seeds were washed in $1 \%$ sodium under sterile conditions for $24 \mathrm{~h}$. For this assay, PDA-mannitol agar plates, adjusted to an osmotic potential of $1 \mathrm{MPa}$ by adding $74,69 \mathrm{~g} * \mathrm{l}^{-1} \mathrm{D}$-mannitol, were prepared, following the method described in Scandiani et al. (2011). Fungal cultures were grown on PDA-mannitol plates until the colonies spread all over the plates. Surface sterilized seeds were placed on those plates and incubated for $48 \mathrm{~h}$ under growth conditions described for fungal cultures above. The inoculated seeds were planted in ED73 substrate, and seedling growth was monitored. The mock control consisted of surface-sterilized seeds, that were incubated on PDA-mannitol plates without fungus.

\section{Results}

132 Cross-infection assays on soybean and lupin hypocotyls

133 Some plant pathogenic fungi from the genus Colletotrichum are known to have a broad host 134 range and, in addition, their lifestyles can range from necrotrophy to endophytic behavior 135 (Talhinhas et al. 2016; De Silva et al. 2017). In this study, we firstly investigated 136 Colletotrichum species originating from soybean or lupin for their ability to cross-infect both 
hosts. The infection process of Colletotrichum species on host plants is mediated by a specific

infection structure called appressorium. This specialized cell is melanized and facilitate a

direct penetration of the host cuticle and cell wall by weakening the plant tissue with the help

of secreted lytic enzymes in combination with physical pressure driving the entry of the

infection hypha (Bechinger 1999; Küster et al. 2008; Ludwig et al. 2014; Loehrer et al. 2014).

To assess the principal ability of the Colletotrichum isolates under investigation to colonize a

host plant irrespectively of appressorium formation, we firstly employed an infection assay

based on fungus-inoculated toothpicks that are directly placed into the seedling's hypocotyls.

In case of successful colonization, lesions are formed at the site wounding caused by the

toothpicks, and lesion length in comparison to the mock-inoculated control served as a

measure for virulence (Fig. 1a). Different field isolates of $C$. truncatum caused the largest

lesions on hypocotyls of the German soybean cultivar Abelina (maturity group 000). Mean

lesion lengths of the C. truncatum isolates LFN150, LFN152 and LFN154 were 5.1, 5.4 and

$4.8 \mathrm{~mm}$, respectively, and significantly different from the mean lesion length of $1,8 \mathrm{~mm}$

caused by the sterile, non-inoculated toothpick tip alone (Fig. 1a). Infection with isolates of $C$.

plurivorum and $C$. lupini, as well as $C$. higginsianum, also resulted in lesions larger than the 
Lila Baer. Again, the adapted C. lupini isolate BBA70385 caused the most prominent lesions

164 (mean value of $11.46 \mathrm{~mm}$ lesion length - Fig. 1c). With mean lesion length ranging from 2.0

to $3.3 \mathrm{~mm}$, the $C$. truncatum isolates LFN153, LFN152 and LFN154 caused smaller, but still

significant, lesion lengths.

Cross-infection assays using inoculated seeds of soybean and lupin

To assess the cross-infectivity of different Colletotrichum isolates on soybean and lupin in an independent assay, we performed seed inoculation experiments. This assay is assumed to be closer to the natural way of plant infection because the fungus must infect the seedling without previous wounding. The same set of fungal isolates as in the toothpick-inoculation assay was tested on soybean cv. Abelina and narrow-leafed lupin cv. Lila Baer (Fig. 2). Because not all seeds made it to normal-looking seedlings, we chose a classification of five categories encompassing a range of non-emerged seedlings (0), seedlings which emerged but did not develop further (1), and seedlings which are smaller (2), equally (3) or better (4) developed in comparison to the mock control. On soybean, all C. truncatum isolates had severe effects on seedling emergence, in most cases inhibiting emergence. C. plurivorum isolates LFN0007, LFN0010, LFN0018, LFN0023, the $C$. lupini isolate, and the C. higginsianum isolate caused retardation in seedling development in comparison to mocktreated seeds one week after sowing.

Interestingly, seeds inoculated with C. plurivorum isolate LFN0019 developed better in comparison to non-inoculated seeds (Fig. 2a). This plant growth-promoting effect of $C$. plurivorum was even more prominent in the seed-inoculation experiment with L. angustifolius cv. Lila Baer (Fig. 2b). Here all but one isolate of C. plurivorum led to seedlings, that developed better than seeds from the non-inoculated control. In this experiment, the $C$. plurivorum isolate LFN0023 led to a similar reduction in seedling development as the $C$. 
lupini and the $C$. higginsianum isolate. All $C$. truncatum isolates clearly impaired lupin seedling development, and seed-inoculation with the $C$. truncatum isolate LFN153 completely prevented seedling emergence (Fig. 2b).

\section{Plant-growth promoting effect on lupin}

The purpose of the seed inoculation experiments was to get an overview of the interactions of the whole range of fungal species and isolates in this study; therefore, they were based on single experiments with a lower number of plants. To support the observation regarding the plant growth-promoting effects, an independent biological experiment with L. angustifolius cv. Lila Baer and the C. plurivorum isolates was performed (Fig. 3). Instead of using our custom rating system, this time, the height of plants was measured, and the developmental stage was assessed according to a lupin-adapted BBCH scale. The plant developed from $C$. plurivorum inoculated seeds all were significantly taller than the plants originating from mock-treated seeds after seven weeks (Fig. 3a). The rating, according to a lupin-adapted $\mathrm{BBCH}$ scale showed that not only plant height was positively affected, but also the number of leaves (Fig. 3b). Whereas the tendency of an increased leaf number could be observed for all interactions with the fungal isolates, only interactions with C. plurivorum isolates LFN0010 and LFN0023 proved to be significantly different from mock-control. Figure 4 shows representative plants from this experiment seven weeks after sowing. The differences between plants of the non-inoculated control and those emerged from seeds treated with the fungus are easily visible (Fig. 4a). It seems that seedlings are not only taller after inoculation with $C$. plurivorum, rather the development as a whole is affected as some of the plants already progressed to flowering stage (Fig. 4b). 


\section{Discussion}

213 In this study, the interaction of Colletotrichum spec. isolates originating from soybean or

214 lupin were tested for the first time on soybean and lupin cultivars actually used in Germany

215 Since, to our knowledge, so far soybean anthracnose has not yet been systematically

216 investigated on soybean in Germany, we made use of isolates of C. truncatum and

217 C. plurivorum coming from Brazil (Rogério et al. 2017; Barbieri et al. 2017).

To get an overview of the virulence spectrum of the different fungal isolates on both host plants, at first, an assay with a simple read-out, namely measurement of lesion lengths, was performed. This method using toothpicks colonized by the fungi was already established and successfully applied for studying Fusarium virguliforme and C. truncatum and C. plurivorum on soybean and applied here for the first time to lupin hypocotyls (Scandiani et al. 2011; lesion length measurement is easily quantifiable. However, the inoculation method including wounding and is thus not closely related to the natural infection process. By wounding, the pre-formed resistance barriers of the cuticle and an intact epidermis are circumvented.

Differences in colonization, therefore, must be attributed to defense mechanisms active during the colonization of the plant in response to the invading fungus (Hutcheson 1998; Scandiani et al. 2011). On soybean cv. Abelina all C. truncatum colonized the hypocotyl beyond the wound-induced lesion, but only the isolates LFN150, LFN152, and LFN154 lead to significant large lesions (Fig 1a). The soybean-derived isolates of $C$. plurivorum, as well as $C$. lupini caused smaller lesions than the $C$. truncatum isolates. Remarkably, also $C$. higginsianum, known to naturally infect plants from the Brassicaceae family was able to invade the tissue to a certain extent albeit also not statistically significant (Fig. 1a). This rather 
237 which possess the general ability to grow on a variety of plants as well as dying and dead

238 tissue (Damm et al. 2012; De Silva et al. 2017).

239 In the toothpick assay, C. lupini caused the largest lesions on both lupin cultivars Amiga and

240 Lila Baer (Fig. 1, b and c). The interactions of lupin with the other Colletotrichum isolates

241 revealed a more differentiated picture between L. albus (Fig. 1, b) and L. angustifolius (Fig. 1,

242 c), which might be attributed to differences in basal resistance of both lupin cultivars.

243 Since anthracnose of soybean and lupin is reported to be seed-born, we decided to also

244 employ a seed infection assay to get closer to the natural infection process (Talhinhas et al.

245 2016; Pecchia et al. 2019). For this assay, seeds were co-incubated with the fungus on agar

246 plates. The swelling of the seeds followed by germination, which quickly happens after

247 placing seeds on agar-medium, would make this method inadequate We prevented swelling

248 and germination by increasing the osmotic potential of the agar-medium, enabling a longer

249 co-incubation phase for seed and fungus, resulting in very evenly inoculated seeds (Scandiani

250 et al. 2011). Soybean seeds pre-treated this way with C. truncatum isolates only rarely

251 developed seedlings, and again, also C. lupini and C. higginsianum showed a tendency to

252 cause retarded seedling development (Fig 2, a). In the case of L. angustifolius cv. Lila Baer,

253 the C. truncatum isolate LFN153 exhibited a stronger negative effect on seedling emergence,

254 than C. lupini (Fig 2, b). Most interestingly, besides causing retardation of seedling

255 development, some $C$. plurivorum interactions led to promotion of plant growth of both

256 soybean and lupin seedlings (Fig. 2). These findings were confirmed in an independent

257 experiment using L. angustifolius and the C. plurivorum isolates (Fig. 3). In addition to

258 evaluation in the first experiment, the development of plants was monitored over a longer

259 time span. After five weeks the plant growth-promoting effect could be observed for all

260 interactions tested. This was not only manifested by increased plant height (Fig 3, a), but also

261 by the advancement to more mature developmental stages as observed in comparison to plants 
262 growing from mock-treated seeds (Fig. 3, b). Thus, after seven weeks, some of the inoculated 263 plants already progressed to flowering stage (Fig. 4.).

264 In contrast to the first experiment, the isolate LFN0023 also caused a plant growth-promoting 265 effect. Our results underline the plasticity of the interaction of Colletotrichum spec. with 266 different hosts which can range from pathogenicity to commensalism and mutualism. Maybe 267 also external factors influence this balance, as a substrate poor in nutrients was used in the second experiment and plants were grown longer than in the first experiment. It is known, that Colletotrichum species exhibit a broad spectrum of different lifestyles ranging from endophytic growth to necrotrophic behavior and that dynamic transitions in lifestyle can happen depending on host plants and environmental factors (Hacquard et al. 2016; Hiruma et al. 2016; De Silva et al. 2017).

Our study revealed, that soybean-derived isolates of Colletotrichum spec. are capable of causing disease on lupin and that $C$. lupini, at least under laboratory conditions, can colonize soybean. Whether or not this may cause problems in legume production in Germany cannot be foreseen, but considering the overlap of areas where soybean and lupin production is

277 possible, this might only be a matter of time (Fig. 5). In this study, we reported on a plant growth-promoting effect of fungi from the genus Colletotrichum for legumes; however, the underlying mechanism remains enigmatic. Future studies have to explore the mechanism and should address the question and whether the fungus can grow endophytically like, e.g., Piriformospora indica (syn. Serendipita indica) (Deshmukh et al. 2006; Zuccaro et al. 2011). approaches in legume production. 


\section{Author contributions}

287 ML and US conceived and planned the experiments. Louisa Wirtz carried out the

288 experiments, processed experimental data and drafted figures. ML analyzed the data, finalized

289 the figures and drafted the manuscript. RRL and NSMJ conceived and adapted the inoculation

290 assays on soybean, provided material and contributed to interpretation of results. BRW

291 encouraged to study anthracnose on lupin, provided material and gave critical feedback on the

292 results. All authors read and approved the final manuscript.

294 Compliance with Ethical Standards: The authors declare that they have no conflict of

295 interest. No experiments involving humans or animals have been conducted.

297 Acknowledgments

298 The authors are grateful to Monika Hermanns for technical assistance. Many thanks to

299 Richard O’Connell for kindly providing the C. higginsianum isolate.

\section{References}

302

303

304

Barbieri MCG, Ciampi-Guillardi M, Moraes SRG, et al (2017) First Report of Colletotrichum plurivorum Causing Anthracnose on Soybean in Brazil. Plant Dis 101:1677-1677. doi: 10.1094/PDIS-07-16-0963-PDN

Bechinger C (1999) Optical Measurements of Invasive Forces Exerted by Appressoria of a Plant Pathogenic Fungus. Science 285:1896-1899. doi: 10.1126/science.285.5435.1896

Berger JD, Buirchell BJ, Luckett DJ, Nelson MN (2012) Domestication bottlenecks limit genetic diversity and constrain adaptation in narrow-leafed lupin (Lupinus angustifolius 
Damm U, Cannon PF, Crous PW (2012) Colletotrichum: complex species or species complexes?

Damm U, Sato T, Alizadeh A, et al (2019) The Colletotrichum dracaenophilum, C. magnum and C. orchidearum species complexes. Stud Mycol 92:1-46. doi:

Deshmukh S, Huckelhoven R, Schafer P, et al (2006) The root endophytic fungus Piriformospora indica requires host cell death for proliferation during mutualistic symbiosis with barley. Proc Natl Acad Sci 103:18450-18457. doi: 10.1073/pnas.0605697103

Dias MD, Fonseca MEN, Dias-Neto JJ, et al (2018) Biology, pathogenicity, and haplotype analyses of Colletotrichum plurivorum: a novel soybean anthracnose agent in warm tropical areas. Trop Plant Pathol 43:439-451. doi: 10.1007/s40858-018-0249-6

Fischer K, Dieterich R, Nelson MN, et al (2015) Characterization and mapping of LanrBo: a locus conferring anthracnose resistance in narrow-leafed lupin (Lupinus angustifolius L.). Theor Appl Genet 128:2121-2130. doi: 10.1007/s00122-015-2572-3

Hacquard S, Kracher B, Hiruma K, et al (2016) Survival trade-offs in plant roots during colonization by closely related beneficial and pathogenic fungi. Nat Commun 7:11362. doi: $10.1038 /$ ncomms 11362

Hiruma K, Gerlach N, Sacristán S, et al (2016) Root Endophyte Colletotrichum tofieldiae 

Confers Plant Fitness Benefits that Are Phosphate Status Dependent. Cell 165:464-474. doi: 10.1016/j.cell.2016.02.028 Rev Phytopathol 36:59-90. doi: 10.1146/annurev.phyto.36.1.59

Jacob I, Feuerstein U, Heinz M, et al (2017) Evaluation of new breeding lines of white lupin with improved resistance to anthracnose. Euphytica 213:1-10. doi: 10.1007/s10681-017-

Küster S, Ludwig N, Willers G, et al (2008) Thin PTFE-like membranes allow characterizing germination and mechanical penetration competence of pathogenic fungi. Acta Biomater

Murphy-Bokern D, Stoddard FL, Watson CA (2017) Legumes in cropping systems. Legum

$$
\text { 4:1809-1818. doi: 10.1016/j.actbio.2008.05.011 }
$$

Loehrer M, Botterweck J, Jahnke J, et al (2014) In vivo assessment by Mach-Zehnder doublebeam interferometry of the invasive force exerted by the Asian soybean rust fungus (Phakopsora pachyrhizi). New Phytol 203:620-631. doi: 10.1111/nph.12784

Lucas MM, Stoddard FL, Annicchiarico P, et al (2015) The future of lupin as a protein crop in Europe. Front Plant Sci 6:1-6. doi: 10.3389/fpls.2015.00705

Ludwig N, Loehrer M, Hempel M, et al (2014) Melanin Is Not Required for Turgor Generation but Enhances Cell-Wall Rigidity in Appressoria of the Corn Pathogen Colletotrichum graminicola. Mol Plant-Microbe Interact 27:315-327. doi: 10.1094/MPMI-09-13-0267-R

Nirenberg HI, Feiler U, Hagedorn G (2002) Description of Colletotrichum lupini comb. nov. in modern terms. Mycologia 94:307-320. doi: 10.1080/15572536.2003.11833238 
Pecchia S, Caggiano B, Da Lio D, et al (2019) Molecular Detection of the Seed-Borne Pathogen Colletotrichum lupini Targeting the Hyper-Variable IGS Region of the Ribosomal Cluster. Plants 8:222. doi: 10.3390/plants8070222

Ploper LD, Backman PA (1992) Nature and Management of Fungal Diseases Affecting Soybean Stems, Pods, and Seeds. In: Pest Management in Soybean. Springer Netherlands, Dordrecht, pp 174-184

Rogério F, Ciampi-Guillardi M, Barbieri MCG, et al (2017) Phylogeny and variability of Colletotrichum truncatum associated with soybean anthracnose in Brazil. J Appl Microbiol 122:402-415. doi: 10.1111/jam.13346

Rogério F, Gladieux P, Massola NS, Ciampi-Guillardi M (2019) Multiple Introductions Without Admixture of Colletotrichum truncatum Associated with Soybean Anthracnose in Brazil. Phytopathology 109:681-689. doi: 10.1094/PHYTO-08-18-0321-R

Scandiani MM, Ruberti DS, Giorda LM, et al (2011) Comparison of inoculation methods for characterizing relative aggressiveness of two soybean sudden-death syndrome pathogens, Fusarium virguliforme and F. tucumaniae. Trop Plant Pathol 36:133-140. doi: $10.1590 /$ S1982-56762011000300001

Talhinhas P, Baroncelli R, Le Floch G (2016) Anthracnose of lupins caused by Colletotrichum lupini: A recent disease and a successful worldwide pathogen. J Plant Pathol 98:5-14. doi: 10.4454/JPP.V98I1.040

Tian Q, Lu C, Wang S, et al (2017) Rapid diagnosis of soybean anthracnose caused by Colletotrichum truncatum using a loop-mediated isothermal amplification (LAMP) assay. Eur J Plant Pathol 148:785-793. doi: 10.1007/s10658-016-1132-2

Zuccaro A, Lahrmann U, Güldener U, et al (2011) Endophytic life strategies decoded by genome and transcriptome analyses of the mutualistic root symbiont Piriformospora 
indica. PLoS Pathog 7:e1002290. doi: 10.1371/journal.ppat.1002290

\section{$381 \quad$ Figure captions}

382 Fig. 1 Results of lesion length measurements after inoculation of soybean and lupin 383 hypocotyls

Hypocotyls of G. max cv. Abelina (a), L. albus cv. Amiga (b) and L. angustifolius cv. Lila

Baer (c) were infected with Colletotrichum-inoculated toothpick tips and lesion lengths were measured after seven days. The abbreviations " $C t$ ", " $C p$ ", " $C l$ ", and " $C h$ " stand for the species C. truncatum, C. plurivorum, C. lupini, and C. higginsianum, respectively. Bars represent the mean value of two to four independent biological experiments with two plants each. Error bars show standard deviation. Asterisks indicate a statistically significant difference in comparison to mock control, determined by a one-way ANOVA analysis on ranks, followed by a Dunn's posthoc test $(\mathrm{P}<0.05)$. In the graphs, the dashed line represents the mean value of the mock control.

Fig. 2 Rating of seedling emergence and development after inoculation of soybean and lupin seeds with Colletotrichum isolates

Seeds of G. max cv. Abelina (a) and L. angustifolius cv. Lila Baer plants (b) were inoculated with different Colletotrichum species and rated one week after sowing. The abbreviations " $C t$ ", " $C p$ ", " $C l$ ", and " $C h$ " stand for the species C. truncatum, C. plurivorum, C. lupini, and C. higginsianum, respectively. The following categories for rating were applied: 0 - seedling not emerged; 1 - seedling development arrested after emergence; 2 - slower or impaired seedling development in comparison to seedlings emerging from mock-inoculated seeds; 3 
403

404

405

406

407

408

409

410

411

412

413

414

415

416

417

418

419

420

421

422

423

424 Fig. 5 Comparison of potential cultivated area for narrow-leafed sweet lupin and soybean in

represent the mean value of five plants and error bars show standard deviation. In both graphs, the dashed line represents the mean value of the mock control.

Fig. 3 Plant-growth promoting effect of C. plurivorum inoculation on lupin development

In an independent experiment, heights of L. angustifolius cv. Lila Baer plants after inoculation of seeds with different $C$. plurivorum isolates were measured five weeks after sowing (a). The plants were also scored according to a lupin-adapted BBCH development scale (b). Bars represent the mean value of at least five plants and error bars show standard deviation.

Statistical significance in relation to mock was tested with a one-way ANOVA analysis followed by Dunnett's $(\mathrm{P}<0.05$, number sign) or Holm-Sidak $(\mathrm{P}<0.05$, asterisk) posthoc test (a). In b) test for statistical significance was performed by a one-way ANOVA analysis on ranks, followed by a Dunn's posthoc test $(\mathrm{P}<0.05$, asterisk). In both graphs, the dashed line represents the mean value of the mock control.

Fig. 4 Plant-growth promoting effect of Colletotrichum plurivorum in seed inoculation assay

Comparison of plants from L. angustifolius cv. Lila Baer after treatment of seed without (a, left side) or with C. plurivorum isolate LFN0010 (a, right side) seven weeks after sowing. Part b) and c) of the figure are enlargements of the respective white dashed boxes in a), indicated by black arrows. White arrows in b) point to developing flowers. Pictures correspond to data shown in Fig. 3.

425 Germany 
426 Possible areas for cultivation of narrow-leafed sweet lupin (a, vertical hatching) and soybean

427 (b, vertical hatching) based on soil-climate-areas are illustrated. An overlay of a) and b)

428 shows the overlap of areas (c, gray-underlaid cross-hatching). Data for maps were obtained

429 and modified from Julius Kühn-Institut (geoportal.julius-kuehn.de/index, accessed 08/2019)

430 under license according to German GeoNutzV and from German Federal Agency for

431 Cartography and Geodesy (http://www.bkg.bund.de, accessed 08/2019) under Data license

432 Germany - attribution - Version 2.0 (dl-de/by-2-0). 
abioRxiv preprint doi: https://doi.org/10.1101/2020.06.19.160853; this version posted June 20,2020. The copyright holder for this preprint

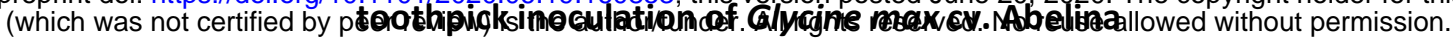

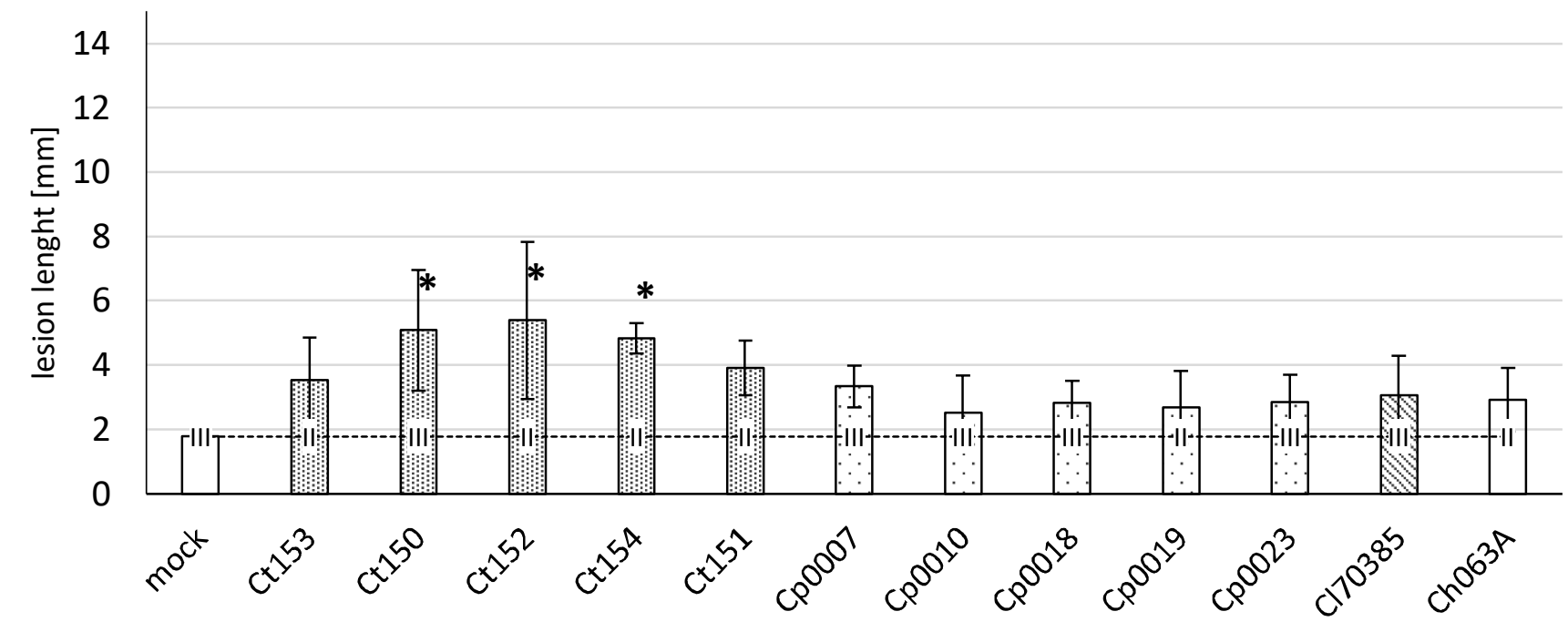

b

\section{toothpick inoculation of Lupinus albus cv. Amiga}

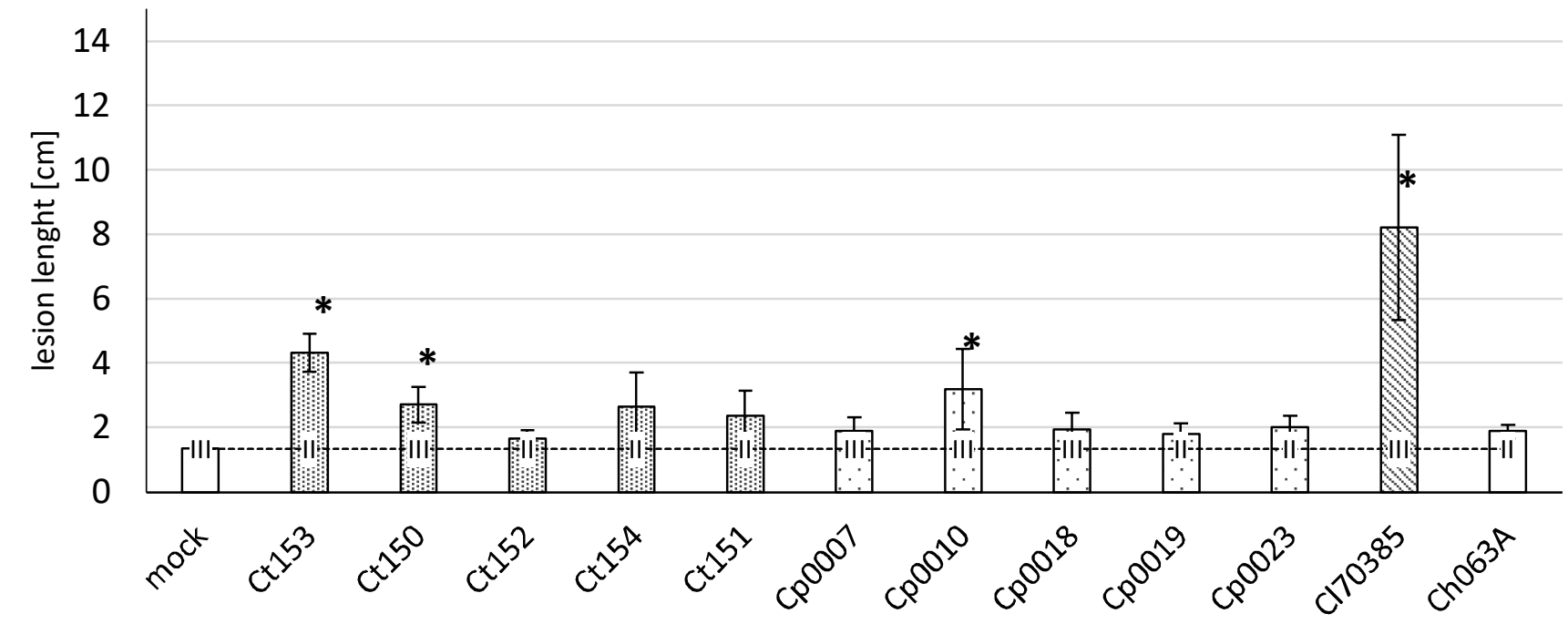

C

toothpick inoculation of Lupinus angustifolius cv. Lila Baer

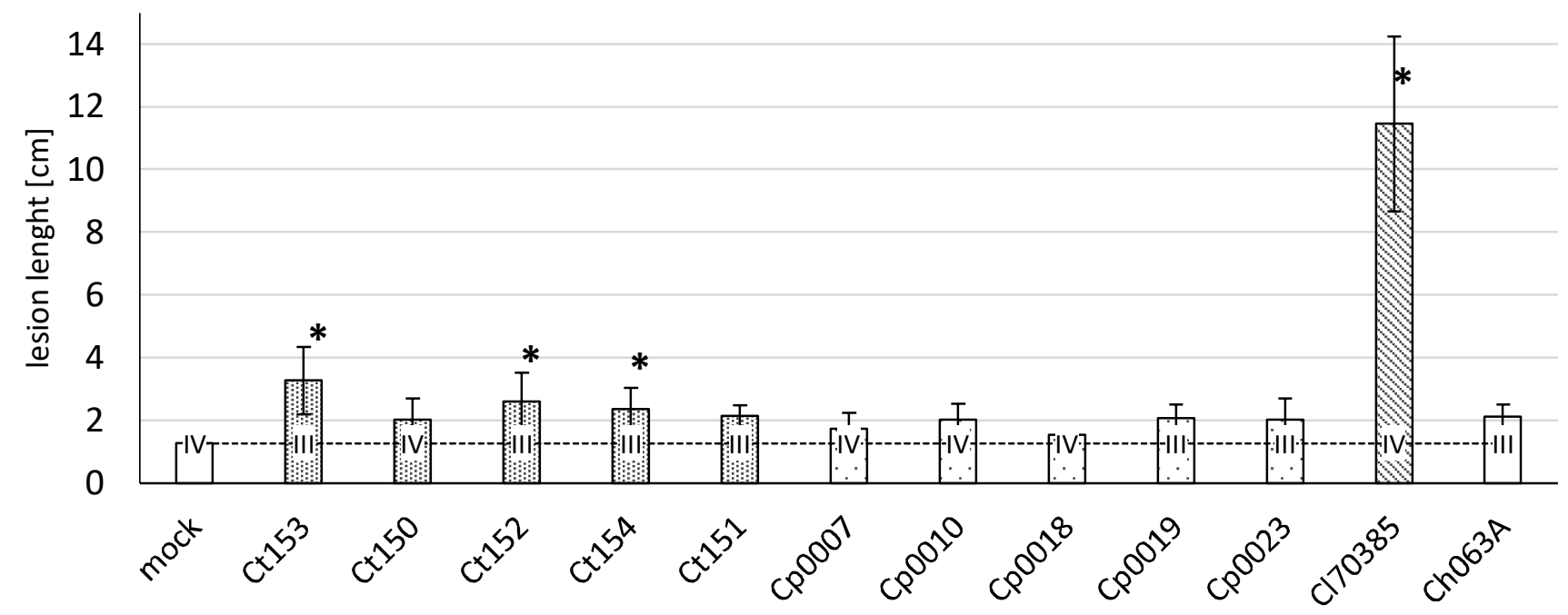

Fig. 1 


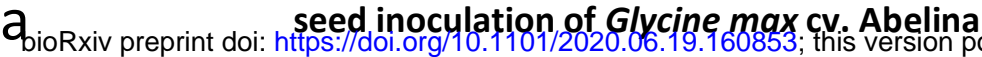

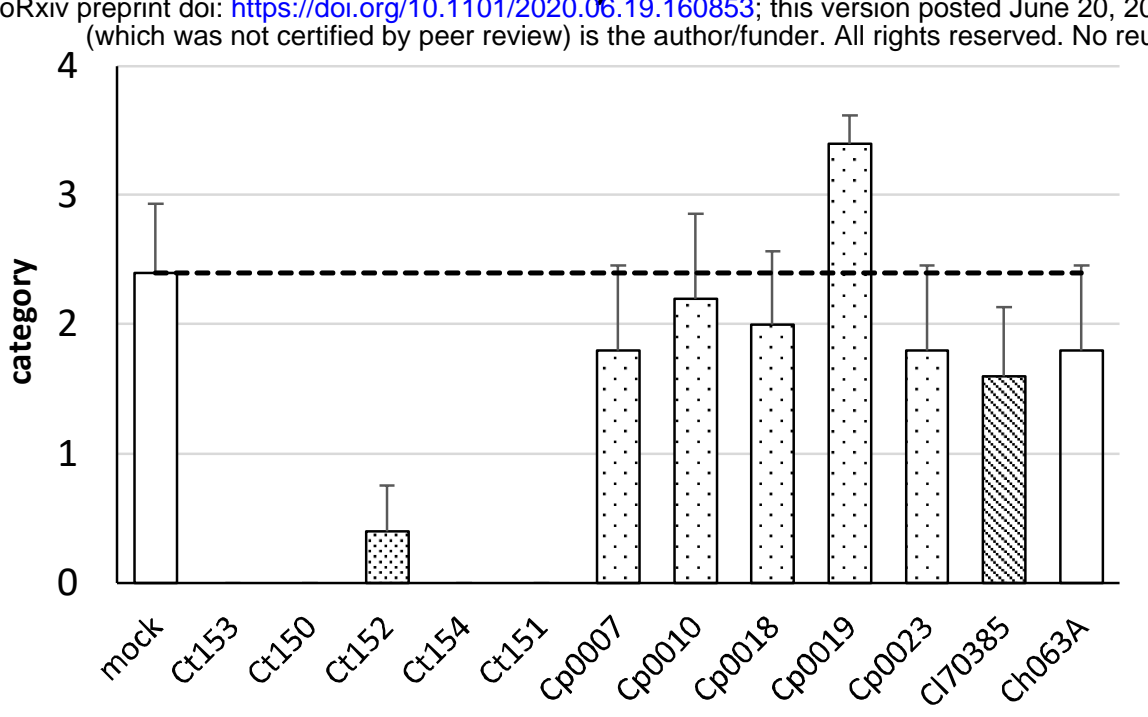

b

seed inoculation of L. angustifolius cv. Lila Baer

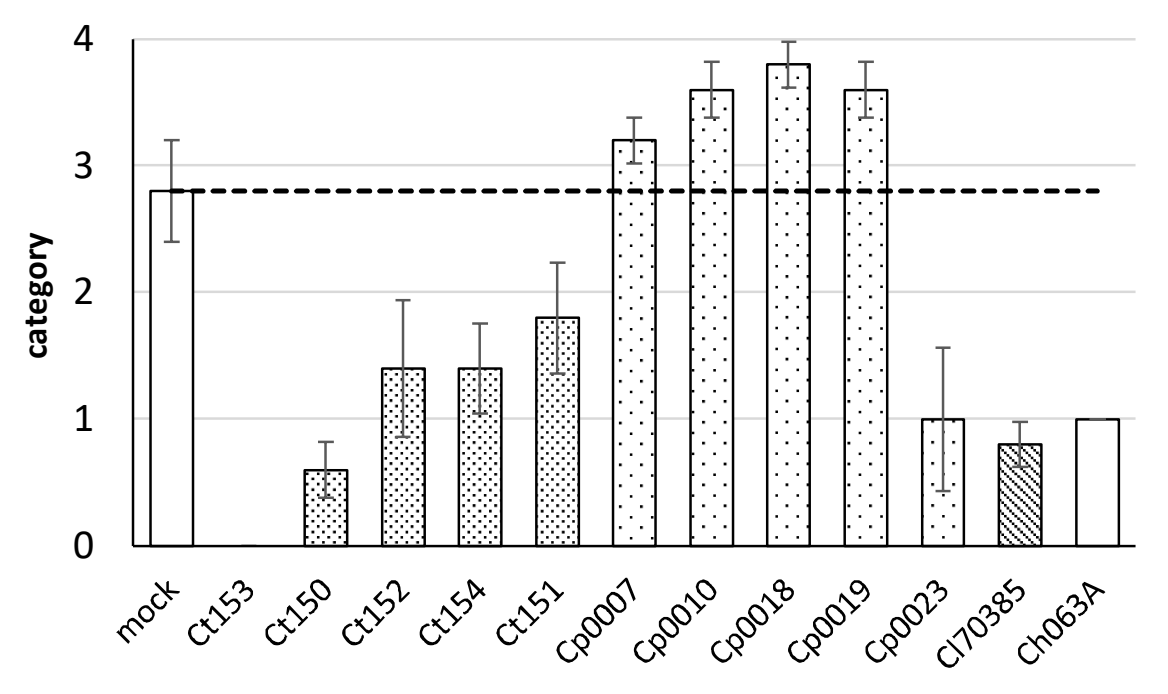

Fig. 2 
bioRxiv preprint doi: https://doi.org/19.1101/2020 2619.160853 this versign posted fune 20 2020. The copyright holder for this preprint

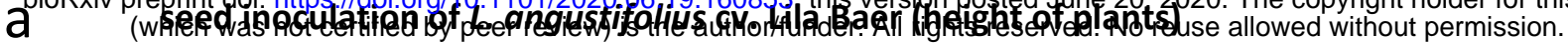

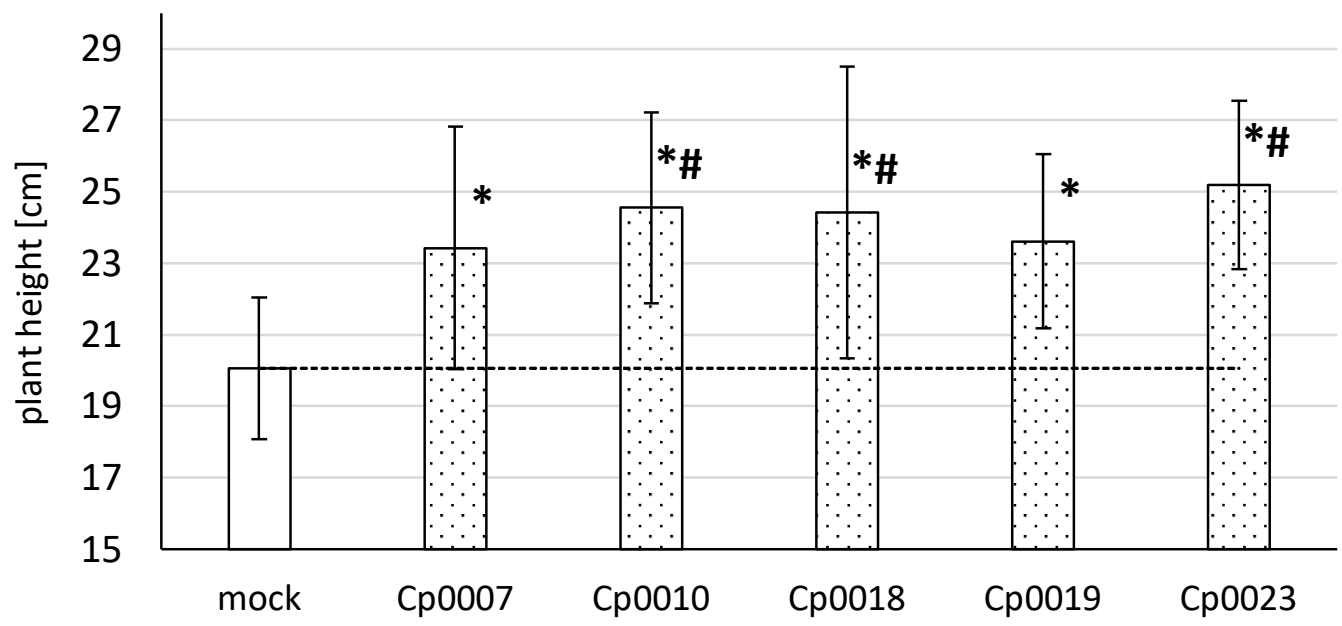

b seed inoculation of $L$. angustifolius cv. Lila Baer (BBCH stage)

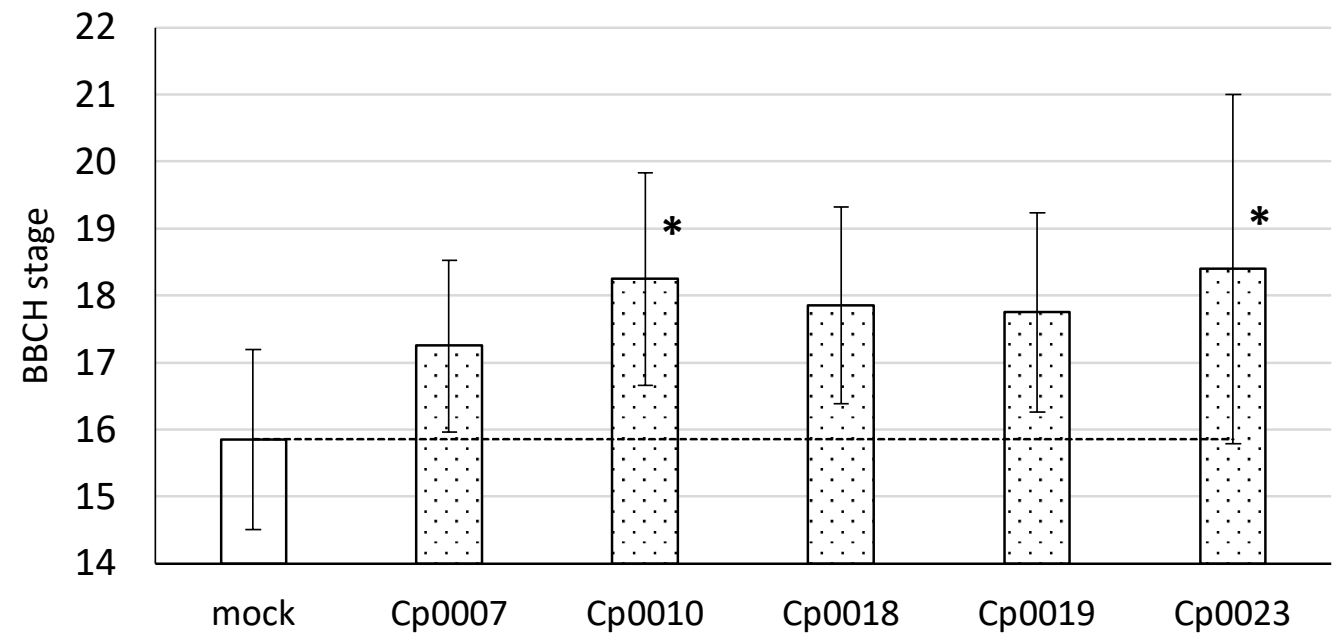

Fig. 3 


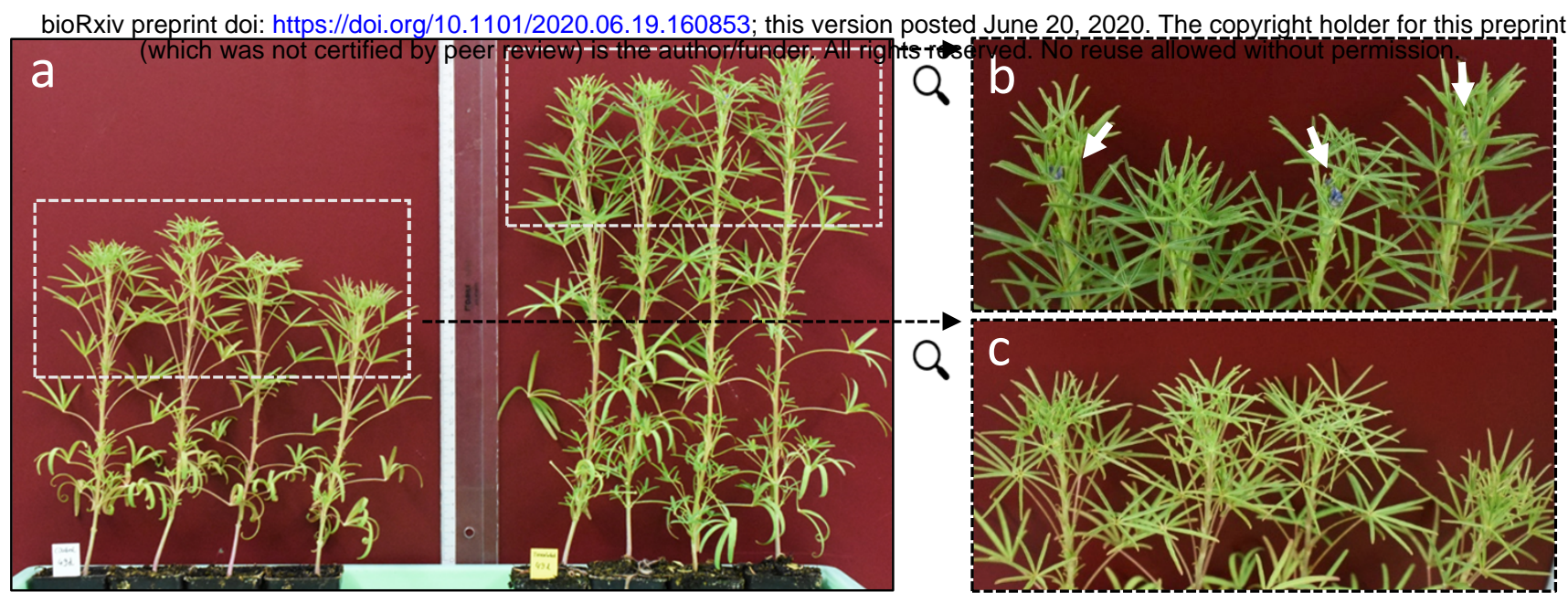

Fig. 4 
bioRxiv preprint doi: https://doi.org/10.1101/2020.06.19.160853; this version posted June 20, 2020. The copyright holder for this preprint

a

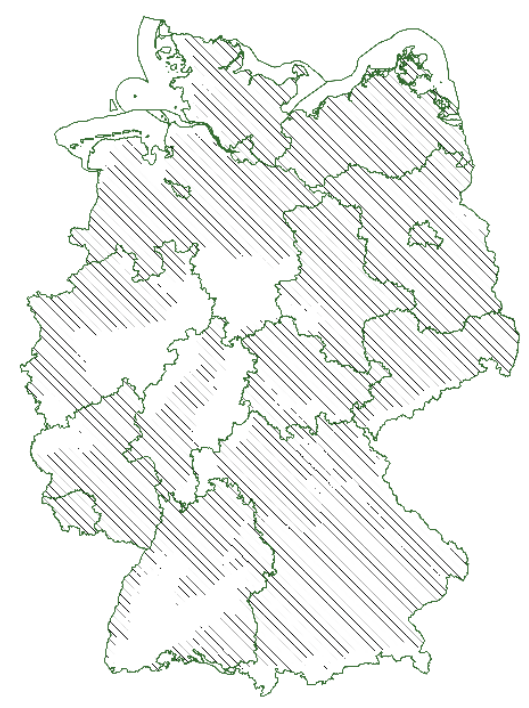

b

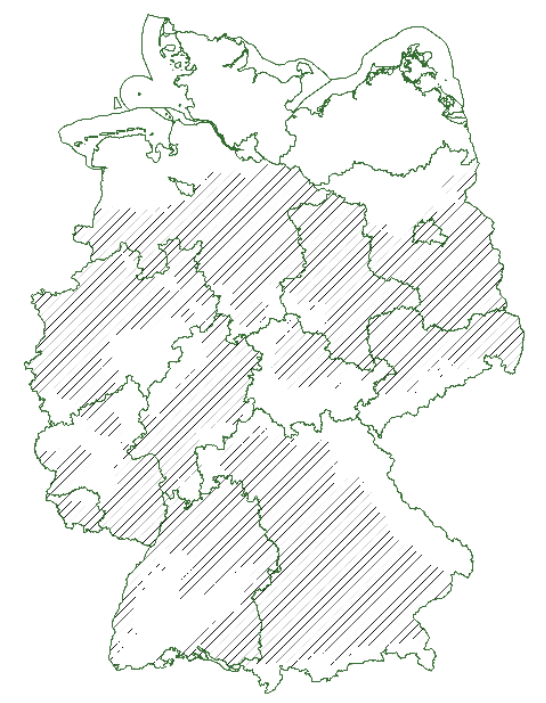

C

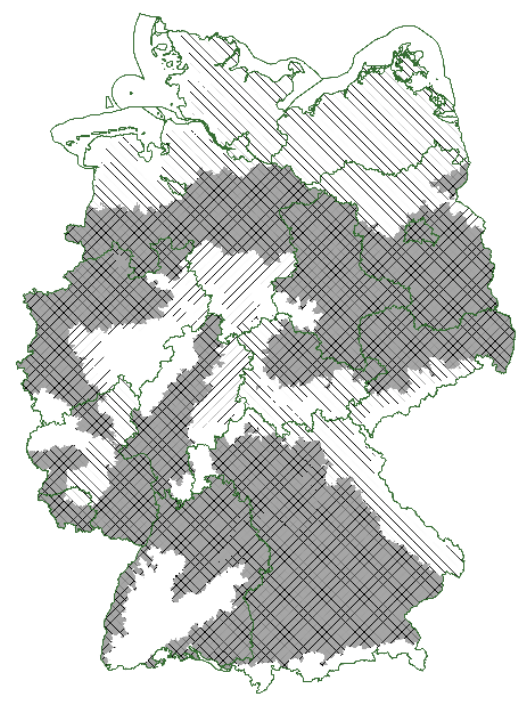

Fig. 5 